\title{
Silkworm Pupae Protect Against Alzheimer's Disease
}

\author{
${ }^{1,2}$ Jintanaporn Wattanathorn, ${ }^{1,2}$ Supaporn Muchimapura, \\ ${ }^{3}$ Artit Boosel, ${ }^{4}$ Sombat Kongpa, ${ }^{4}$ Wiroje Kaewrueng, \\ ${ }^{1,2}$ Terdthai Tong-Un, ${ }^{1,2}$ Panakaporn Wannanon and ${ }^{1,2}$ Wipawee Thukhammee \\ ${ }^{1}$ Department of Physiology, Faculty of Medicine, \\ ${ }^{2}$ Integrative Complimentary and Alternative Medicine Research Group, \\ ${ }^{3}$ Department of Physiology and Graduate School (Neuroscience Program), \\ Khon Kaen University, Khon Kaen, 40002, Thailand \\ ${ }^{4}$ The Queen Sirikit Department of Sericulture, \\ Ministry of Agriculture and Cooperatives, Chatuchak, Bangkok, 10900, Thailand
}

Received 2012-04-06, Revised 2012-06-20; Accepted 2012-08-16

\begin{abstract}
Silkworm (Bombyx mori) pupae have long been used as food and medicine in Asian countries. It is reputed for the treatment of numerous neurological disorders related to oxidative stress including stroke. Therefore, we hypothesized that silkworm pupae could attenuate memory impairment and neurodegeneration in Alzheimer's disease. In the present study, we determined the effect of silkworm pupae on the neurodegeneration and memory impairment in animal model of Alzheimer's disease. Adult male Wistar rats, weighing 180-220 g, were orally given the silkworm pupae at doses of 60,90 and $135 \mathrm{mg} \mathrm{kg}^{-1} \mathrm{BW} 14$ days before and 7 days after the bilateral administration of AF64A, a cholinotoxin, via intracerebroventricular route. The animals were determined the memory using Morris water maze test and determined the density of neurons in hippocampus. All doses of silkworm pupae used in this study significantly mitigated the memory impairment and the decreased neurons density in hippocampus. To explore the possible underlying mechanism of the cognitive enhancing effect and neuroprotective effect, the activity of acetylcholinesterase enzyme and the Malondialdehyde (MDA), the oxidative marker were determined respectively. Our results clearly demonstrated that the cognitive enhancing effect of silkworm pupae occurred at least via the increased cholinergic function while its neuroprotective effect occurred via the decrease oxidative stress. In conclusion, silkworm pupae appear to be the potential functional food to protect against Alzheimer's disease.
\end{abstract}

Keywords: Silkworm Pupae, Protects Against, Alzheimer's Disease, Bilateral Administration, Cognitive Enhancing, Memory Impairment, Clearly Demonstrated

\section{INTRODUCTION}

Alzheimer's disease has been considered to be the most widespread variety of dementia. The etiology of this condition is not completely understood but it has been recognized as the multiple etiologies disorder. Numerous factors have been proposed to contribute the important role on the pathophysiology of Alzheimer's Disease (AD). Recent findings demonstrated the attribution of oxidative stress in various disorders including AD (Pratico and Delanty, 2000; Markesbery, 1999). It was found that level of free radicals oxidation of lipids, proteins and DNA were elevated in postmortem brain of AD (Tuppo and Forman, 2001). Moreover, the decreased cholinergic function and degeneration of cholinergic neurons were also observed (Liston et al., 2004; Zarotsky et al., 2003; Perry et al., 1999). Despite the most commonly dementia found in the elderly and the increasing prevalence, the therapeutic strategy against AD is still very limited. Most of the drugs used nowadays usually produce side effects and can only slow down the progression of diseases. Therefore, the novel potential strategy is required. 
Silkworm or Bombyx mori L. has been long term used as food and medicine in Asian countries. According to the Traditional Chinese Medicine, it is believed that silkworm could promote Chi or the body life energy while balancing the nervous system. Moreover, it has been consumed as heath food especially for cardiac and diabetic patients, bronchial asthma, primary trigeminal neuralgia, facial palsy, pain vocal nodules and polyps. In addition, it also possessed antijuvenoid (Saha et al., 2007), immune booster (Zhang et al., 2006), antioxidant (Kwon et al., 2006) and estrogenic effects (Yang et al., 2010). Previous studies demonstrated that silkworm pupae are a source of proteins and substances which are essential for the function of the nervous system such as vitamin B 1, B 2 and E (Singh and Jayasomu, 2002). Based on the role of oxidative stress in the pathophysiology of $\mathrm{AD}$, its reputation to balance life energy, the antioxidant activity and its effect on the nervous system, we hypothesized that $B$. mori might be able to mitigate the memory impairment and neurodegeneration in AD. Therefore, this study was undertaken to determine the effect of $B$. mori on the memory impairment and neurodegeneration in animal model of AD.

\section{MATERIALS AND METHODS}

\subsection{Animals}

Adult male Wistar rats (180-220 g, 8 weeks old) were obtained from National Animal Center, Salaya, Nakhon Pathom and they were housed in group of 5 per cage in standard metal cages at $22 \pm 2^{\circ} \mathrm{C}$ on $12: 12 \mathrm{~h}$ light-dark cycle. All animals were given access to food and water ad libitum. The experiments were performed to minimize animal suffering in accordance with the internationally accepted principles for laboratory use and care of European Community (EEC directive of 1986; 86/609/EEC). The experimental protocols were approved by the Institutional Animal Care and Use Committee.

\subsection{Preparation of Bombyx mori Powder}

The male silk Bombyx mori pupae state 5 were authenticated by Wiroje Kaewrueng, the Queen Sirikit Institute of Sericulture, Ministry of Agrictulture and Cooperative, Thailand. The voucher specimen was also kept there. The B.mori pupae were isolated from their cocoon, dried at $60^{\circ} \mathrm{C}$ at a period of 4 days. After being dried, the weight of $\mathrm{B}$ mori pupae was decreased to $20 \%$ of wet weight. The dried B.mori pupae were grounded to powder, then separately packed (100 grams/pack) and kept in the cool dry place until use. The suspension of B.mori pupae was freshly prepared to a desired concentration by using Propylene Glycol (PG) as vehicle. In order to provide the feeding convenient, B.mori powder was suspended in a suspending agent of PG.

\subsection{Drugs and Chemicals}

Donepezil hydrochloride (Aricept $10 \mathrm{mg} /$ tablet) (Pfizer pharmaceuticals Inc.) was used as standard drugs in this study. They were dissolved in propylene glycol and administered via oral route. All chemical substances used in this study were analytical grade.

\subsection{Experimental Protocol}

All rats were randomly assigned to 6 groups of 8 animals each.

\subsection{Group I}

Vehicle + ACSF Rats had been treated with propylene glycol which served as vehicle to suspend the B.mori for 2 weeks before and 1 week after the administration of Artificial Cerebrospinal Fluid (ACSF), a vehicle of AF64A.

\subsection{Group II}

Vehicle + AF64A Rats had been treated with vehicle for 2 weeks before and 1 week after the administration of AF64A, a cholinotoxin, in order to induce a cholinergic deficit as found in Alzheimer's disease.

\subsection{Group III}

Donepezil+AF64A: Animals were treated with donepezil, a cholinesterase inhibitor which used as standard drug for dementia treatment that served as positive control in this study, as same as that mentioned in group II.

\subsection{Group IV}

VI B.mori+AF64A: Rats had been treated with the plant extract at various doses ranging from 60, 90 and $135 \mathrm{mg} \mathrm{kg}^{-1} \mathrm{BW}$ for 2 weeks before and 1 week after the administration of AF64A respectively (The doses used in this study were selected based on our preliminary data on the cognitive enhancing effect).

The animals were determined the spatial memory 1 week after AF64A administration. Then, they were sacrificed and determined the density of survival neurons and in various subregions of hippocampus.

\subsection{AF64A Administration}

AF64A was prepared as an aqueous solution of acetylethylcholine mustard $\mathrm{HCl}$ (Sigma, St. Louis, MO) was adjusted to $\mathrm{pH} 11.3$ with $\mathrm{NaOH}$. After stirring for 30 min at room temperature, the $\mathrm{pH}$ was lowered to 7.4 with the gradual addition of $\mathrm{HCl}$ and stirred for $60 \mathrm{~min}$. The amount of AF64A was then adjusted either to $2 \mathrm{nmol} / 2$ $\mu \mathrm{L}$. The vehicle of AF64A was distilled water prepared in the same manner as the AF64A and recognized as ACSF. 
In order to administer AF64A bilaterally via intracerebroventricular (i.c.v.) route, the animals were anesthetized with the intraperitoneal injection of sodium pentobarbital at dose of $60 \mathrm{mg} \mathrm{kg} \mathrm{BW}{ }^{-1}$. Then, AF64A (2 $\mathrm{nmol} / 2 \mu \mathrm{L})$ was infused bilaterally via intracerebroventricular (i.c.v.) route with a 30 -gauge needle inserted through a burr hole drilled into the skull into both the right and left lateral ventricles. Stereotaxic coordinates were (from the bregma): posterior $0.8 \mathrm{~mm}$, lateral $\pm 1.5 \mathrm{~mm}$ and ventral (from dura) $3.6 \mathrm{~mm}$. The rate of infusion was $1.0 \mu \mathrm{L} \mathrm{min}{ }^{-1}$ and the needle was left inplace for $5 \mathrm{~min}$ after infusion and then slowly withdrawn.

\subsection{Morris Water Maze Test}

The Morris water maze test is one of the most important paradigms used for testing spatial navigation task, which is thought to be dependent on the proper functioning of the hippocampus. The testing apparatus for all task used in this study was a stainless steel circular pool that $147 \mathrm{~cm}$ in diameter and $47 \mathrm{~cm}$ in dept. The interior of the pool was flat and the pool was placed on the steady floor. The pool was filled with water to a depth of $12 \mathrm{~cm}$. The water were maintained at $23 \pm 1^{\circ} \mathrm{C}$ and darkened by nontoxic powder.

The pool was divided into four quadrants (NE, NW, SE and SW) by two imaginary lines crossing the center of the pool. For each animal, the invisible platform was placed in the center of one of the quadrants and was remained there for a training period of 4 days. Each rat was gently placed in the water facing the wall of the pool from one of the four starting points $(\mathrm{N}, \mathrm{E}, \mathrm{S}$ or $\mathrm{W})$ along the perimeter of the pool and the animal was allowed to swim until it climbed onto the platform. When an animal could not reach the platform in $60 \mathrm{~s}$, it was gently placed on the platform by the experimenter. In either case, the animal was left on the platform for $10 \mathrm{~s}$ and removed from the pool. Then, it was quickly dried with a towel before being returned to the home cage. The behavior of the experimental animal such as latency to finding the platform, total distance traveled, time spent in the target quadrant of the pool were recorded.

\subsection{Histological Procedure}

Following anesthesia with sodium pentobarbital (60 $\mathrm{mg} \mathrm{kg}^{-1} \mathrm{BW}$ ), fixation of the brain was carried out by transcardial perfusion with fixative solution containing $4 \%$ paraformaldehyde in $0.1 \mathrm{M}$ phosphate buffer $\mathrm{pH} 7.3$. The brains were removed after perfusion and stored over a night in a fixative solution that used for perfusion. Then, they were infiltrated with $30 \%$ sucrose solution for approximately $4{ }^{\circ} \mathrm{C}$. The specimens were frozen rapidly and $30 \mu \mathrm{M}$ thick sections were cut on cryostat. They were rinsed in the phosphate buffer and picked up on slides coated with $0.01 \%$ of aqueous solution of a high molecular weight poly L-lysine.

\subsection{Choline Acetyltransferase Immunohistochemistry}

and

A series of rat brain sections containing hippocampus from each groups were reacted in parallel experiments using a mouse monoclonal antibody detected against Choline Acetyltransferase (ChAT) (Chemicon Internation, Inc., CA, USA) and a modification of a previously described protocol employing the DAKO Strept ABC Complex/HRP duet kit. In brief, rat brain sections were eliminated endogenous peroxidase activity by $0.5 \% \mathrm{H}_{2} \mathrm{O}_{2}$ in methanol. Rat brain sections were washed in running tap water and distilled water for $1 \mathrm{~min}$ each, then rinsed in KPBS and KPBS-BT for $5 \mathrm{~min}$ per each process. Excess was removed, then, the rat brain sections were incubated for $30 \mathrm{~min}$ in a blocking solution composed of 5\% normal horse serum in KPBS-BT. The rat brain sections were then incubated in mouse primary antibody against ChAT diluted $1: 100$ in KPBS$\mathrm{BT}$ at room temperature for $2 \mathrm{~h}$ and then incubated at $4^{\circ} \mathrm{C}$ for $48 \mathrm{~h}$. The tissue was rinsed in KPBS-BT (two washes $\times 7 \mathrm{~min}$ ), incubated for $4 \mathrm{~h}$ in biotinylated goat antimouse IgG antibody, rinsed in KPBS-BT (two washes $\times 7 \mathrm{~min}$ ) and then incubated in Strep ABCComplex/HRP for $4 \mathrm{~h}$. In preparation for visualization step, rat brain sections were rinsed in KPBS-BT $(1 \mathrm{~min})$ and KPBS (two washes $\times 10$ min). ChAT immunoreactivity was visualized using $0.025 \% 3,3$ ' diaminobenzidine (DAB, Sigma) and $0.01 \%$ $\mathrm{H} 2 \mathrm{O} 2$. Finally, rat brain sections were rinsed in running tap water, air dried and cover-slipped using permount.

\subsection{Morphological Analysis}

Five coronal rat brain sections in each group were studied quantitatively. Neuronal counts in hippocampus were performed by eye using a 40x magnification with final field $255 \mu^{2}$ according to the following stereotaxic coordinates: AP $-4.8 \mathrm{~mm}$, lateral $\pm 2.4-6 \mathrm{~mm}$, depth 3-8 $\mathrm{mm}$. The observer was blind to the treatment at the time of analysis. Viable stained neurons were identified on the basis of a stained soma with at least two visible processes. Counts were made in five adjacent fields and the mean number extrapolated to give total number of neurons per $255 \mu^{2}$. All data are represented as number of neurons per $255 \mu \mathrm{m}^{2}$.

\subsection{Acetylcholinesterase and Malondialdehyde Assays}

The rats were divided into various groups as previously described in 2.3. After the last dose of administration, all rats were sacrificed. Hippocampus was isolated, prepared as hippocampal homogenate and the determination of the Malondialdehyde (MDA) level and acetylcholinesterase activity in hippocampus were performed. Malondialdehyde was indirectly estimated by determining the accumulation of Thiobarbituric Acid Reactive Substances (TBARS) in the hippocampal 
homogenate whereas acetylcholinesterase activity was performed using the colorimethod.

\subsection{Statistical Analysis}

Data are presented as mean \pm Standard Error of Mean (SEM). One-way Analysis of Variance (ANOVA), followed by Tukey post hoc test. A probability level less than 0.05 was accepted as significance.

\section{RESULTS}

\subsection{The Effect of B.mori on Spatial Memory}

The effect of B.mori on spatial memory was determined using the validated test, Morris water maze test. The results were shown in Fig. 1. The results clearly revealed that AF64A significantly increased escape latency time (p-value $<0.001$, compared with vehicle+ACSF). Donepezil and all doses of B.mori could reverse the elevation of escape latency induced by AF64A (p-value $<0.001$ all, compared with vehicle+AF 64A). The retention time was evaluated $24 \mathrm{~h}$ after the assessment of escape latency. Figure 2 showed that AF64A significantly decreased retention time ( $\mathrm{p}$-value $<0.01$, compared to vehicle+ACSF). Again, these changes were reversed by donepezil ( $\mathrm{p}$-value $<0.01$, compared to vehicle+AF64A) and all doses of B.mori (p-value < 0.001 all, compared to vehicle+AF64A).

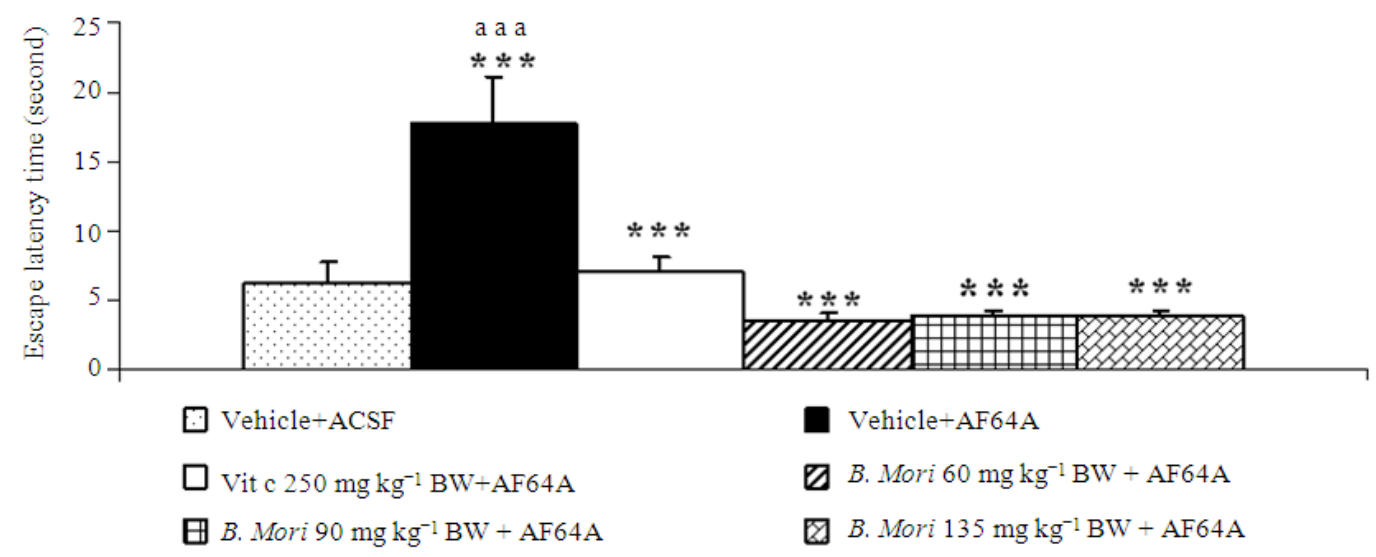

Fig. 1. The effect of B.mori on spatial memory. (Escape latency time)

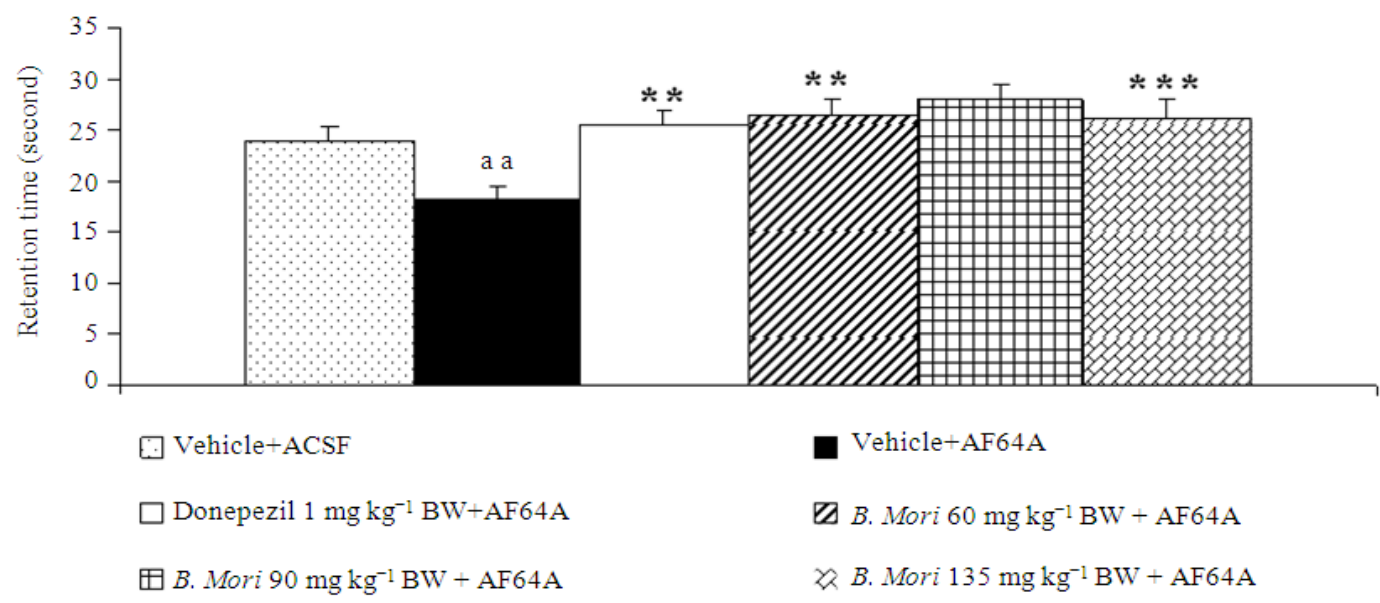

Fig. 2. The effect of B.mori on spatial memory. (Retention time) 


\subsection{The Effect of B.mori on Cholinergic Neurons Density}

Since the spatial memory has been reported to involve the function of cholinergic system and hippocampus, we also determined the effect of B.mori on cholinergic neurons density in hippocampus. Figure 3 showed that AF64A significantly decreased the cholinergic neuron density in CA1, CA2, CA3 and dentate gyrus ( $p$-value $<.001$ all, compared with vehicle $+\mathrm{AF}$ 64A). Donepezil significantly reversed the cholinergic neuron density in CA1, CA2 and dentate gyrus (p-value $<0.05,0.01$ and 0.01 respectively, compared with vehicle+AF64A). It was found that rats subjected to B.mori treatment at dose of $90 \mathrm{mg} \mathrm{kg}^{-1} \mathrm{BW}$ significantly increased cholinergic neuron density in CA1, CA2, CA3 and dentate gyrus (p-value $<0.01,0.01,0.05$ and 0.05 respectively, compared with vehcle+AF64A) while the high dose treatment could enhance the cholinergic neuron density in CA1, CA2 and dentate gyrus ( $p$-value < $0.05,0.05$ and 0.001 respectively, compared with vehicle+AF64A).

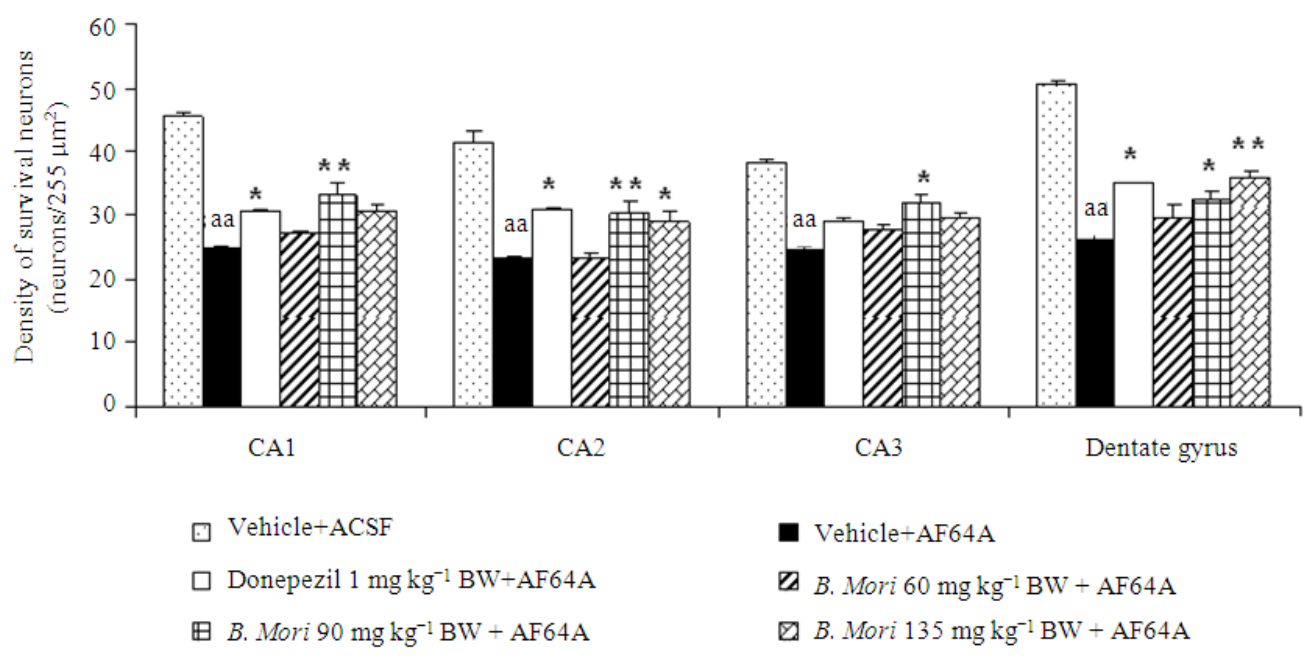

Fig. 3. The effect of B.mori on cholinergic neurons density

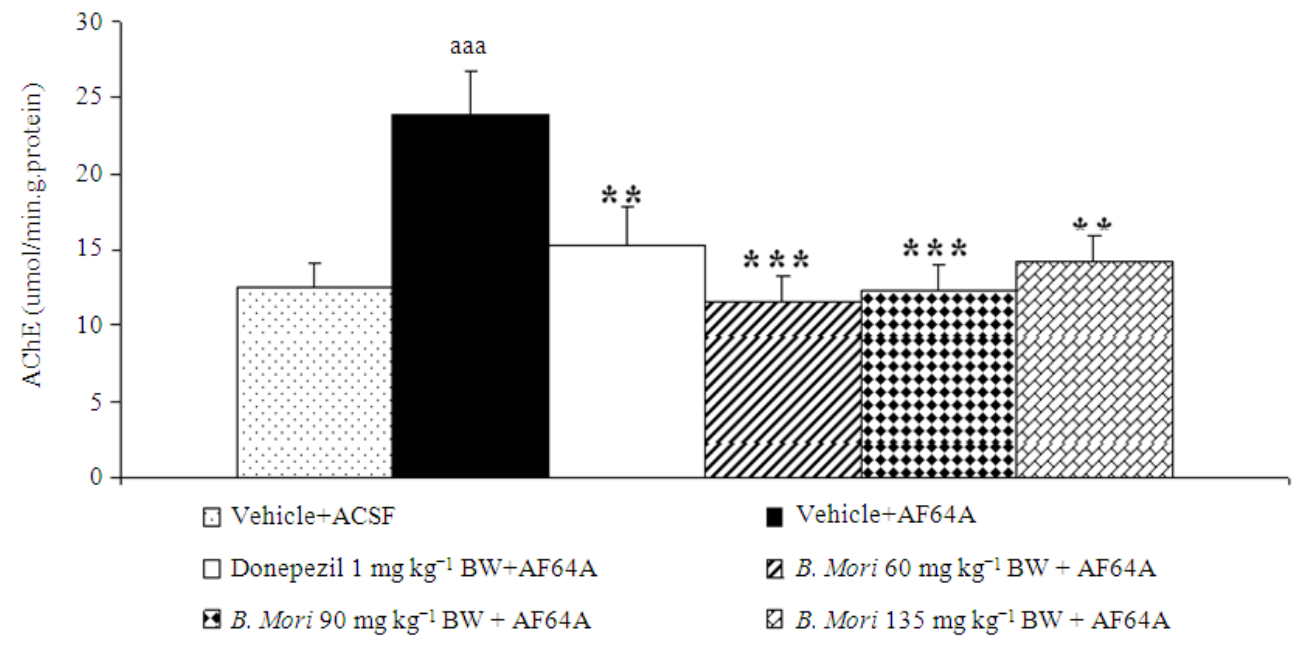

Fig. 4. Effect of B.mori on acetylcholinesterase activity 


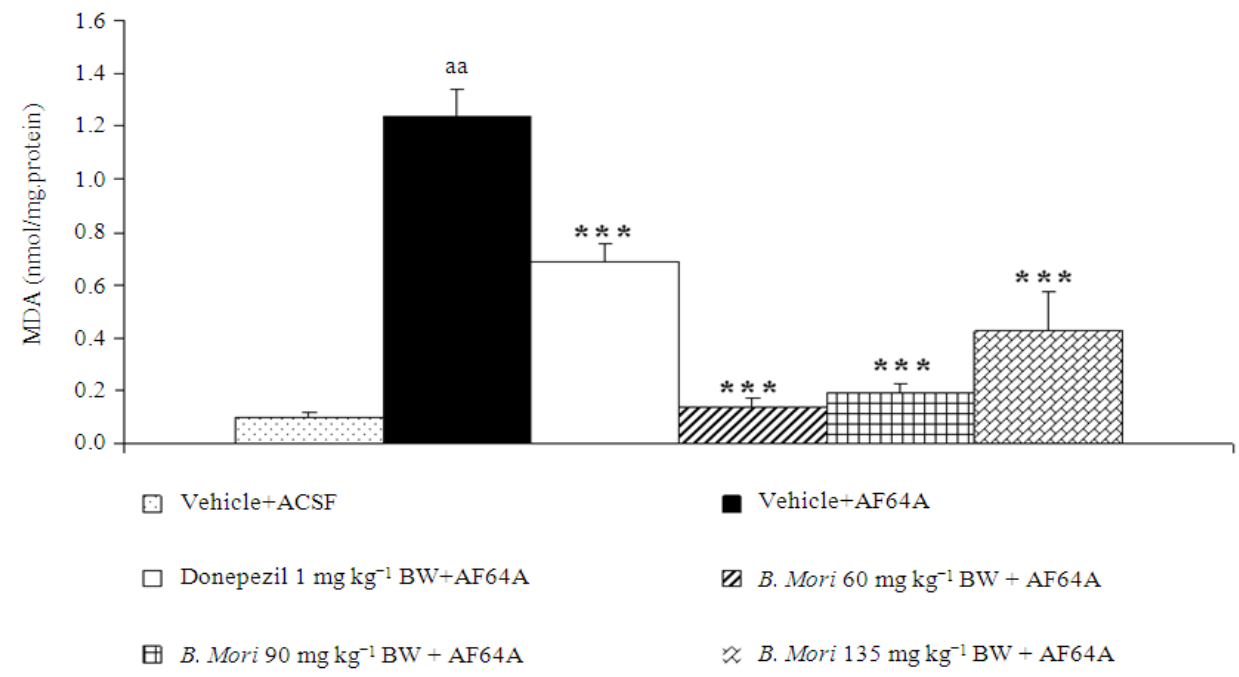

Fig. 5. Effect of B.mori on malondialdehyde level in hippocampus

\subsection{Effect of B.mori on Acetylcholinesterase Activity and Malondialdehyde Level in Hippocampus}

Based on the previous information that Acetylcholinesterase (AChE) indirectly indicated the level of Acetylcholine (ACh), we also determined the activity of AChE in hippocampus. In Fig. 4, it was found that rats subjected to AF64A significantly increased AChE activity in hippocampus (p-value $<0.001$, compared with vehicle+ACSF). Donepezil treated groups significantly reversed the elevation of $\mathrm{AChE}$ induced by AF64A (p-value $<0.01$. compared with vehicle+AF64A). Surprisingly, B.mori treated groups at all dosage range used in this study could reverse the elevation of AChE activity induced by AF64A much more than that observed in donepezil treated group ( $\mathrm{p}$ value $<0.001$ all, compared with vehicle $+A F 64 A$ ).

Due to the crucial role of oxidative damage on neurodegeneration, we also determined the effect of B.mori on the level of Malondialdehyde (MDA) level in hippocampus. The results were shown in Fig. 5. It was demonstrated that AF64A produced a significant elevation of MDA in hippocampus (p-value $<0.001$, compared with vehicle+ACSF). All doses of B.mori also reversed this elevation ( $\mathrm{p}$-value $<0.001$ all, compared with vehicle+AF64A).

\section{DISCUSSION}

In this study, our results showed that $\mathrm{AF} 64 \mathrm{~A}$ treated rats induced the degeneration of cholinergic neurons density in all subregions of hippocampus as those observed in AD. Dietary supplementation with B.mori pupae powder could enhance the cholinergic function by decreasing both the cholinergic neurons density and $\mathrm{AChE}$ activity in hippocampus. In accompany with these changes, the memory impairment was also improved. In addition, B.mori treated group also showed the markedly reduction of MDA level in hippocampus.

It is widely accepted that oxidative stress is implicated in the pathogenesis of neurodegeneration in numerous conditions including Alzheimer's disease (Stewart and Heales, 2003). Previous studies showed that both the degeneration of cholinergic neurons and the decreased ACh were also observed in the brains of patients attack by AD (DeLaGarza, 2003). In addition, it was reported that the level of antioxidant in plasma of $\mathrm{AD}$ patients were decreased in accompany with the cognitive decline. The reduction of antioxidant enzymes in $\mathrm{AD}$ patients gave rise to the inadequate antioxidant enzymatic activities to counteract the increased free radicals. The administration of dietary rich in antioxidant could decrease the risk of AD (Engelhart et al., 2002). Recently, antioxidant has been proposed to be the potential therapeutic against AD (Behl and Moosmann, 2002; Thukham-Mee et al., 2012). Administration of substances possessing antioxidant activity could increase the activity of Superoxide Dismutase (SOD) but decreased MDA level resulting in the attenuation of memory impairment in animal model of Alzheimer's disease (Li et al., 2010; Luo and Huang, 2006). This was in agreement with our results which demonstrated that B.mori, a substance possessing antioxidant activity, could decrease MDA level in hippocampus. The 
reduction of MDA level indicated the improvement of oxidative stress status which in turn resulted in the increased cholinergic neurons density leading to the attenuation of memory impairment.

Previous study also clearly demonstrated that acetylcholinesterase inhibitor could also improve memory impairment in Alzheimer's disease (DeLaGarza, 2003). Therefore, the cognitive enhancing effect of B.mori in this study might occur partly via the inhibition of AChE.

\section{CONCLUSION}

Although the precise underlying mechanism and the details of the active substances in B.mori remained to be discovered, this local food and herbal medicine according to the folklore might offer a novel approach for the prevention of Alzheimer's disease.

\section{ACKNOWLEDGEMENT}

This study was supported by The Queen Sirikit Department of Sericulture, Ministry of Agriculture and Cooperatives and Integrative Complimentary Alternative Medicine, Khon Kaen University, Khon Kaen, Thailand.

\section{REFERENCES}

Behl, C. and B. Moosmann, 2002. Oxidative nerve cell death in Alzheimer's disease and stroke: Antioxidants as neuroprotective compounds. Biol. Chem., 383: 521-536. PMID: 12033440

DeLaGarza, V.W., 2003. Pharmacologic treatment of Alzheimer's disease: An update. Am. Fam. Physician, 68: 1365-1372. PMID: 14567491

Engelhart, M.J., M.I. Geerlings, A. Ruitenberg, J.C.V. Swieten and A. Hofman et al., 2002. Dietary intake of antioxidants and risk of Alzheimer disease. JAMA, 287: 3223-3229. DOI: 10.1001/jama.287.24.3223

Kwon, E.H., M.A. Jung, S.J. Rhee, S.W. Choi and S.H. Cho, 2006. Antioxidant effects and improvement of lipid metabolism of mulberry fruit, mulberry leaves and silkworm powder with different mixing ratios in streptozotocin-induced diabetic rats. Korean J. Nutr., 39: 91-99.

Li, X.H., C.Y. Li, Z.G. Xiang, F. Zhong and Z.Y. Chen et al., 2010. Allicin can reduce neuronal death and ameliorate the spatial memory impairment in Alzheimer's disease models. Neurosciences (Riyadh), 15: 237-243. PMID: 20956919
Liston, D.R., J.A. Nielsen, A. Villalobos, D. Chapin and S.B. Jones et al., 2004. Pharmacology of selective acetylcholinesterase inhibitors: Implications for use in Alzheimer's disease. Eur. J. Pharmacol., 486: 917. PMID: 14751402

Luo, L. and Y.M. Huang, 2006. Effect of resveratrol on the cognitive ability of Alzheimeros mice. Zhong Nan Da Xue Xue Bao Yi Xue Ban., 31: 566-569. PMID: 16951520

Markesbery, W.R., 1999. The role of oxidative stress in Alzheimer disease. Arch. Neurol., 56: 1449-1452.

Perry, E., M. Walker, J. Grace and R. Perry, 1999. Acetylcholine in mind: A neurotransmitter correlate of consciousness? Trends Neurosci., 22: 273-280. PMID: 10354606

Pratico, D. and N. Delanty, 2000. Oxidative injury in diseases of the central nervous system: Focus on alzheimer's disease. Am. J. Med., 109: 577-585. DOI: 10.1016/S0002-9343(00)00547-7

Saha, A.K., T.D. Biswas, S.K. Das and N.B. Kar, 2007. Antijuvenoid action of terpenoid imidazole compound on the larval-pupal-adult development of silkworm, Bombyx mori L. Int. J. Indust Entomol., 14: 127-135.

Singh, K.P. and R.S. Jayasomu, 2002. Bombyx mori-A review of its potential as a medicinal insect. Pharm. Biol., 40: 28-32.

Stewart, V.C. and S.J.R. Heales, 2003. Nitric oxideinduced mitochondrial dysfunction: Implications for neurodegeneration. Free Radic. Biol. Med., 34: 287303. PMID: 12543245, DOI: 10.1016/S08915849(02)01327-8

Thukham-Mee, W., J. Wattanathorn, S. Muchimapura and C. Bunchonglikitkul, 2012. Neuroprotective effect against Alzheimer's disease of porcine brain extract. Am. J. Applied Sci., 9: 700-708. DOI: 10.3844/ajassp.2012.700.708

Tuppo, E.E. and L.J. Forman, 2001. Free radical oxidative damage and Alzheimer's disease. J. Am. Osteopath. Assoc., 101: 11S-15S.

Yang, H.J., J.W. Lee S.H. Lee, J.S. Ryu and D.H. Kwak et al., 2010. Estrogenic activity produced by aqueous extracts of silkworm (Bombyx mori) pupae in ovariectomized rats. Am. J. Chin. Med., 38: 8997. PMID: 20128047

Zarotsky, V., J.J. Sramek and N.R. Cutler, 2003. Galantamine hydrobromide: An agent for Alzheimer's disease. Am. J. Health Syst. Pharm., 60: 446-452. PMID: 12635450

Zhang, Y.J., Z.L.V. Chen, Z. Nie, X. Zhang and X. Wu, 2006. Can $29 \mathrm{kDa}$ rhGM-CSF expressed by Silkworm pupae bioreactor bring into effect as active cytokine through orally administration? Eur. J. Pharm. Sci., 28: 212-235. DOI: 10.1016/j.ejps.2006.02.014 